13

\title{
Экстракция ионов из полярного раствора с использованием поверхностно-модифицированного мембранного интерфейса
}

\author{
(C) А.А. Балакин, С.Г. Хидиров \\ Филиал Федерального исследовательского центра химической фризики им. Н.Н. Семенова РАН, \\ 142432 Черноголовка, Московская обл., Россия \\ e-mail: balakin@binep.ac.ru
}

Поступило в Редакцию 29 декабря 2018 г.

В окончательной редакции 29 декабря 2018 г.

Принято к публикации 17 июня 2019 г.

Продемонстрирована возможность полевой экстракции ионов из полярного раствора с использованием в качестве интерфейса полимерной трековой мембраны с проводящим покрытием. Получен времяпролетный спектр ионов, экстрагированных из раствора КI в водно-глицериновой смеси наносекундным импульсом электрического поля. Применение проводящего покрытия позволило существенно понизить напряжение экстрагирующего импульса и уменьшить влияние вторичных процессов на состав ионов, генерируемых в импульсном мембранном ионном источнике.

Ключевые слова: полевая экстракция ионов, трековая мембрана, полярный раствор.

DOI: $10.21883 /$ JTF.2019.12.48500.452-18

\section{Введение}

В масс-спектральном анализе растворов полевая экстракция ионов является ключевым процессом в методиках генерации газофазных ионов слаболетучих веществ. Этот процесс используется как метод мягкой ионизации молекул различной природы, поскольку переход ионов из раствора в газовую фазу не сопровождается разрушением исходной структуры имеющихся в растворе ионов. Эффективный переход ионов из полярной жидкости в паровую фазу наблюдается в электрических полях напряженностью порядка $10 \mathrm{MV} / \mathrm{cm}$ и более $[1,2]$. В столь сильном электрическом поле свободная поверхность жидкости не является устойчивой и происходит ее разбрызгивание.

В $[3,4]$ была продемонстрирована возможность создания условий для прямого полевого испарения ионов из полярного раствора без его разбрызгивания. Для стабилизации поверхности использовались каналы полимерной трековой мембраны. Содержащий ионы раствор помещали на поверхность трековой мембраны толщиной около десяти микрон. При этом раствор заполняет каналы мембраны, но не вытекает на другую сторону, что возможно при диаметре каналов в несколько десятков нанометров. Такой мембранный интерфейс позволяет иметь раствор при атмосферных условиях, тогда как с другой стороны мембраны может поддерживаться вакуум. Выход ионов из каналов стимулируется электрическим полем, которое создается за счет того, что между электродом, погруженным в раствор, и электродом, находящимся вблизи мембраны с вакуумной стороны, подается постоянное напряжение. В дальнейшем было показано, что в этих условиях сильное локальное электрическое поле, способное стимулировать достаточно эффективный выход ионов из полярной жидкости, созда- валось благодаря зарядке поверхности диэлектрической мембраны [1,5]. Было установлено, что использование постоянного напряжения имеет ряд существенных недостатков, ограничивающих ее использование, в частности, в аналитических приложениях.

В [6] было предложено использовать для экстракции ионов импульсное электрическое поле. Сильное электрическое поле у поверхности раствора, заполняющего каналы мембраны, благодаря большой разнице в электропроводности раствора и полимерной основы мембраны, обеспечивается за счет эффекта острия. В таком режиме напряженность электрического поля будет пропорциональна напряжению между раствором и экстрагирующим электродом, что, в отличие от стационарного режима, делает выход ионов легко управляемым.

Экспериментальная проверка показала, что стабильную экстракцию ионов из водно-глицериновой смеси можно получить, прикладывая импульсы высокого напряжения - более $5 \mathrm{kV}$ при расстоянии между мембраной и электродом в вакууме около $0.2 \mathrm{~mm}$ [7]. Оказалось, что такая схема экстракции имеет существенный недостаток: в генерируемом источником ионном пучке, наряду с ионами, выходящими из раствора, в большом количестве присутствуют посторонние ионы. Они возникают в процессах вторичной эмиссии при бомбардировке мембраны и электрода в вакууме экстрагируемыми из раствора первичными ионами, ускоренными до энергии в несколько килоэлектронвольт.

Решить проблему влияния вторичных процессов можно существенно уменьшив амплитуду импульсного напряжения, сохранив при этом напряженность электрического поля у поверхности раствора, заполняющего каналы мембраны. Поскольку уменьшение зазора между мембраной и электродом в вакууме в конструкции, 
использовавшейся в [7], вряд ли возможно, было предложено экстрагирующий электрод перенести на вакуумную поверхность мембраны. Оценки параметров такого модифицированного мембранного интерфейса были проведены в [8].

В настоящей работе в качестве первого шага в реализации идеи создания низковольтного мембранного интерфейса была применена полимерная трековая мембрана со сплошным металлическим покрытием на вакуумной стороне. Цель настоящей работы состоит в том, чтобы экспериментально продемонстрировать возможность экстракции ионов из полярного раствора низковольтными импульсами при использовании модифицированного мембранного интерфейса.

\section{Методика эксперимента}

Конструкция использовавшегося интерфейса схематично показана на рис. 1. На металлический диск $1 \mathrm{c}$ отверстием диаметром $3 \mathrm{~mm}$ наклеена лавсановая трековая мембрана 2. На атмосферной стороне расположен полый цилиндрический электрод 3 из нержавеющей стали диаметром $4 \mathrm{~mm}$, с нижней стороны затянутый плоской сеткой с ячейками $0.3-0.35 \mathrm{~mm}$, прилегающей к мембране. С помощью кольцевой резиновой прокладки4, уплотняемой фторопластовой шайбой 5, электрод изолировался от металлического диска с мембраной. С вакуумной стороны на мембрану наносилось проводящее покрытие 6. Исследуемый раствор 7 помещался с атмосферной стороны внутри цилиндрического электрода.

Проводящее покрытие лавсановой трековой мембраны, предварительно наклеенной на металлический диск с отверстием 1 , создавалось путем нанесения методом вакуумного испарения слоя золота толщиной несколько десятых долей микрона. Толщина мембраны около $10 \mu \mathrm{m}$, плотность каналов $-10^{7} \mathrm{~cm}^{-2}$, диаметр каналов, определенный из измерений воздушного потока через сухую мембрану - около $50 \mathrm{~nm}$. Заметного изменения

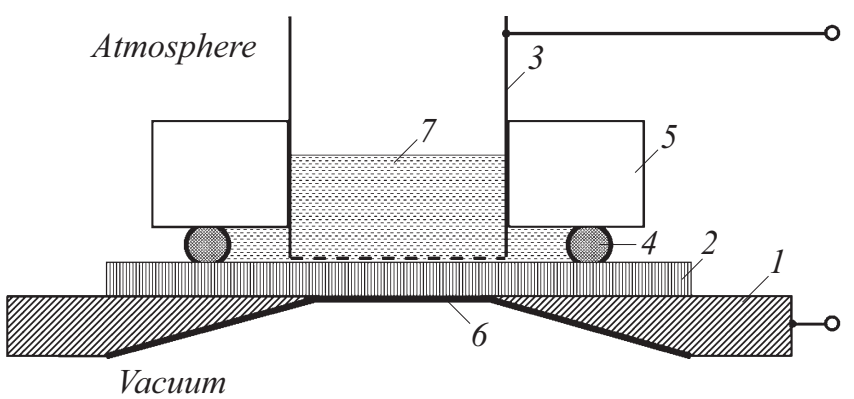

Рис. 1. Поверхностно-модифицированный мембранный интерфейс для транспорта ионов из раствора в вакуум: 1 металлический диск с отверстием; 2 - мембрана; 3 - цилиндрический электрод с сеткой; 4 - изолирующая прокладка; 5 - уплотняющая шайба; 6 - проводящее покрытие; 7 исследуемый раствор.

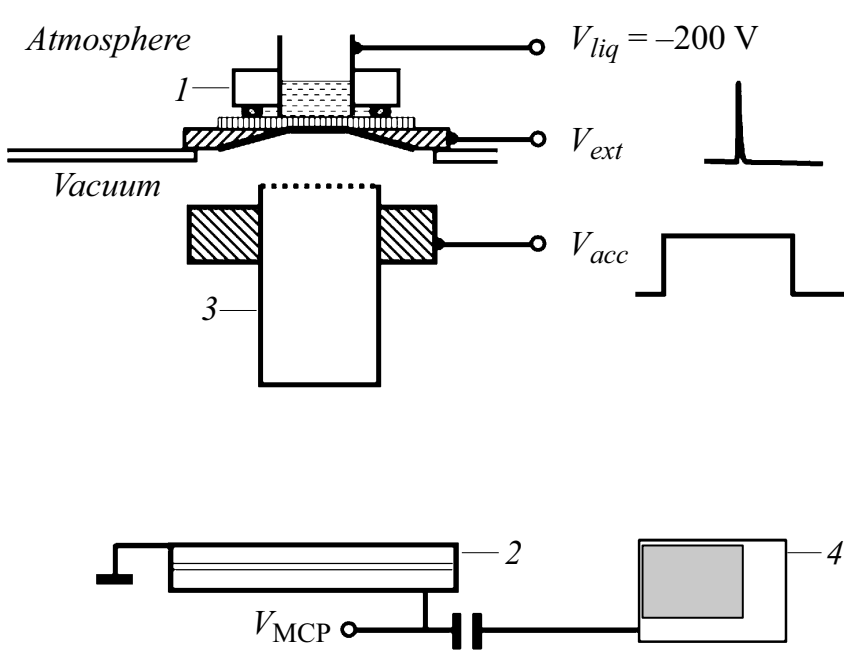

Рис. 2. Схема регистрации отрицательных ионов: 1 - ионный источник; 2 - МСР детектор; 3 - ускоряющий электрод; 4 осциллограф.

диаметра каналов после напыления слоя золота обнаружено не было, поскольку воздушный поток через сухую мембрану оставался практически таким же, как и до напыления.

На электрод с раствором подавалось постоянное напряжение $-200 \mathrm{~V}$. Для экстракции ионов на металлический диск, имеющий электрический контакт с проводящим покрытием на вакуумной стороне мембраны, подавался наносекундный импульс амплитудой до $500 \mathrm{~V}$.

Для регистрации ионов, извлекаемых из каналов мембраны, использовали дрейфовую вакуумную камеру, описанную в [7]. Схема регистрации ионов, экстрагированных из раствора, приведена на рис. 2. В верхней части камеры располагался ионный источник с мембранным интерфейсом 1. В нижней части находился детектор ионов 2, собранный на двух микроканальных пластинах $(\mathrm{MCP})$. Полное расстояние между мембраной и входной поверхностью детектора, находящейся при нулевом потенциале, составляло $24.5 \mathrm{~cm}$.

Для ускорения вышедших из раствора ионов до энергии, необходимой для работы детектора, использовался цилиндрический полый электрод 3 диаметром $16 \mathrm{~mm}$ и длиной $12 \mathrm{~cm}$, затянутый в верхней части металлической плоской сеткой $(1 \times 1 \mathrm{~mm})$. Расстояние между сеткой и мембраной составляло $2 \mathrm{~cm}$. Сигнал с детектора записывался цифровым осциллографом WR 6051 (LeCroy) 4.

Длительность импульса на ускоряющем электроде выбиралась таким образом, чтобы обеспечить свободное движение ионов до попадания на детектор. Это достигалось тем, что экстрагированные из раствора ионы после прохождения сетки оставались внутри электрода в нулевом электрическом поле до окончания импульса ускорения.

Экстрагирующий ионы импульс создавался на резисторе $200 \Omega$ за счет разрядки конденсатора емкостью $180 \mathrm{pF}$, заряжаемого постоянным напряжением, через 


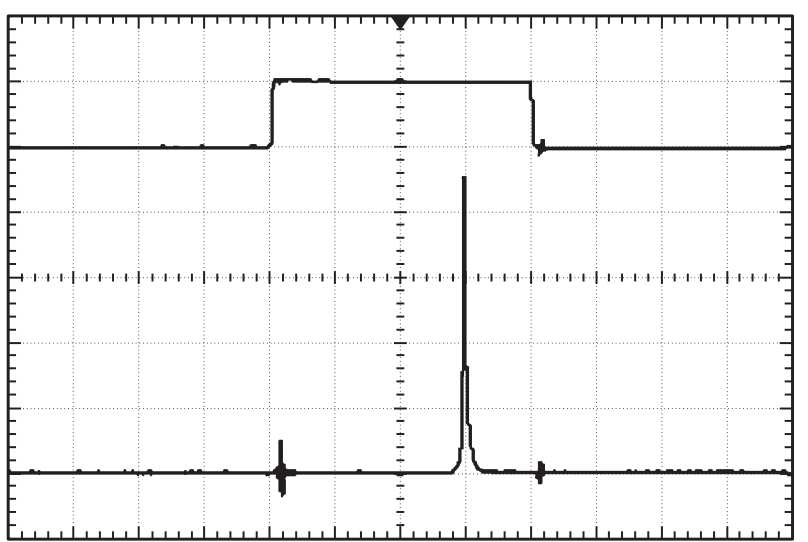

Рис. 3. Осциллограммы напряжения экстрагирующего (внизу) и ускоряющего (вверху) импульсов. Развертка по горизонтали $-1 \mu \mathrm{s} / \mathrm{div}$.

симистор ВТА12-800. Ширина импульса на полувысоте составляла около $20 \mathrm{~ns}$.

Подаваемое на ускоряющий электрод импульсное напряжение амплитудой $3.5 \mathrm{kV}$ генерировалось переключателем PHVSW-005V (Willamate High Voltage).

Система синхронизации и задержки позволяла выбирать относительное положение импульсов экстракции и ускорения. Частота повторения импульсов составляла $100 \mathrm{~Hz}$. На рис. 3 показаны осциллограммы и взаимное положение этих импульсов при экстракции отрицательно заряженных ионов.

Измерения проводились при комнатной температуре.

\section{Результаты и их обсуждение}

В связи с тем, что число ионов, приходящих на детектор при действии одного экстрагирующего импульса было мало, сигналы от отдельных ионов накапливались и усреднялись в осциллографе в цикле из $10^{3}$ последовательных импульсов экстракции. На рис. 4 показана осциллограмма, полученная при экстракции отрицательных ионов импульсом $500 \mathrm{~V}$ из $5.9 \cdot 10^{-3} \mathrm{M}$ раствора KI в смеси глицерина и воды в объемной пропорции 50/50.

Как видно, в сигнале хорошо различима система пиков, ширина которых на полувысоте около $40 \mathrm{~ns}$. Такая осциллограмма, по сути, является дрейф-спектром, который может быть использован для идентификации ионов, выходящих из раствора под действием экстрагирующих импульсов. Небольшое уширение пиков по сравнению с импульсами экстракции связано, по-видимому, с тем, что регистрируемые ионы имеют некоторое распределение по энергии поступательного движения из-за влияния экстрагирующего импульсного электрического поля, а также из-за частичной декластеризации ионов в электрическом поле после их выхода из раствора.

Время пролета ионов $\tau_{i}$ от мембраны до МСР детектора складывается из времени ускоренного движения до сетки ускоряющего электрода, $\tau_{a c c}$, и времени свободного движения от сетки до детектора, $\tau_{f f}$ :

$$
\tau_{i}=\tau_{a c c}+\tau_{f f},
$$

где $\tau_{a c c}$ и $\tau_{f f}$ определяются соотношениями

$$
\begin{gathered}
\tau_{a c c}=d\left(2 m_{i} / q V\right)^{1 / 2}, \\
\tau_{f f}=(L-d)\left(m_{i} / 2 q V\right)^{1 / 2},
\end{gathered}
$$

в которых $m_{i}-$ масса иона, $q-$ его заряд, $V-$ ускоряющее напряжение, $d-$ расстояние между мембраной и сеткой ускоряющего электрода, $L-$ полное расстояние от мембраны до детектора. В соотношении (2) не учтено действие экстрагирующего импульсного поля, поскольку, как показывают оценки, оно слабо влияет на энергию поступательного движения ионов.

При растворении KI в полярном растворителе за счет электролитической диссоциации образуются отрицательные ионы $\mathrm{I}^{-}$. Естественно ожидать, что именно эти ионы должны превалировать в составе ионов, экстрагированных из раствора. Следует учесть, однако, что при полевой экстракции ионов в паровую фазу в основном переходят не сами ионы, а ионные кластеры вида $\mathrm{I}^{-} \mathrm{G}_{n}$, в состав которых входит несколько молекул глицерина G [5]. Масса таких ионных кластеров

$$
M_{i}(n)=M_{i}+n M_{\mathrm{G}},
$$

где $M_{i}$ - масса отрицательного иона йода, $M_{\mathrm{G}}$ - масса молекулы глицерина, $n-$ число молекул глицерина в кластере. Для $n$ от 0 до 4 массы кластерных ионов составляют: 127, 219, 311, 403 и 495 Da соответственно. Расчет по формулам (1)-(3) для $m_{i}=M_{i}(n)$ с учетом того, что суммарное напряжение, ускоряющее ионы, составляло $3.7 \mathrm{kV}$, приводит к временам выхода ионов: $3.59,4.73,5.63,6.41$ и $7.10 \mu$ s для $n$ соответственно 0,1 , 2,3 и 4.

Рассчитанные значения времен пролета кластеров на основе отрицательных ионов иода находятся в хорошем согласии с временами выхода пиков, показанных на рис. 4. Исключение составляет пик с временем выхода около $4 \mu \mathrm{s}$ (рассчитанная масса иона около $148 \mathrm{Da}$ )

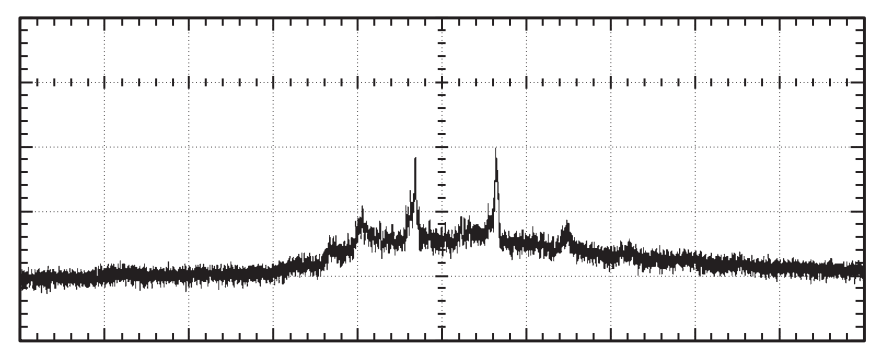

Рис. 4. Осциллограмма сигнала, полученного при экстракции отрицательно заряженных ионов из раствора КI в водноглицериновой смеси. Развертка по времени $-1 \mu \mathrm{s} / \mathrm{div}$, начало развертки соответствует началу импульса экстракции ионов. 


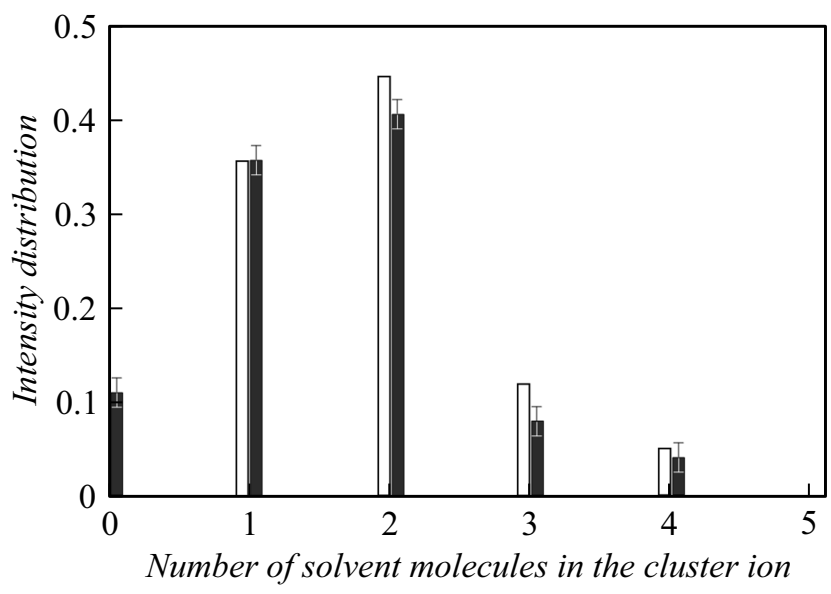

Рис. 5. Диаграмма распределения интенсивностей пиков кластерных ионов иода, экстрагированных из водно-глицериновой смеси наносекундными импульсами (черный) и стационарным электрическим полем (белый).

выпадает из этой серии и связан, возможно, с образованием вторичных ионов при ударе первичных кластерных ионов, экстрагированных из раствора, о сетку ускоряющего электрода.

На рис. 5 для сравнения показано распределение интенсивностей ионных кластеров, зарегистрированных в настоящей работе, и распределения, полученного ранее в [5] при использовании для экстракции ионов стационарного электрического поля. Как видно, в обоих случаях наблюдается довольно широкое распределение ионов по числу молекул растворителя с максимумом для $n=2$. Однако если при экстракции в стационарном поле ион $\mathrm{I}^{-}$без кластерной оболочки не наблюдался, в импульсной методике его вклад заметен.

\section{Заключение}

В работе впервые продемонстрирована возможность полевой экстракции ионов из полярного раствора с использованием в качестве интерфейса полимерной трековой мембраны с проводящим покрытием. Применение бессеточного интерфейса позволило, во-первых, существенно понизить напряжение экстрагирующего импульса (почти в 10 раз по сравнению с методикой, применявшейся ранее). Во-вторых, удалось уменьшить влияние вторичных процессов на состав ионов, генерируемых в импульсном мембранном ионном источнике, что подтвердило предположение о роли вторичных процессов в образовании фоновых ионов в ионном источнике с сеточным экстрагирующим электродом вблизи мембраны.

Таким образом, модифицированный мембранный интерфейс на основе трековой мембраны позволяет получать дрейф-спектры ионов, в которых превалируют ионы, экстрагированные из растворов.

\section{Финансирование работы}

Работ выполнялась в рамках Программы фундаментальных научных исследований государственных академий наук на 2013-2020 годы, комплексная тема ААААA18-118112690060-9.

\section{Конфликт интересов}

Авторы заявляют, что у них нет конфликта интересов.

\section{Список литературы}

[1] Balakin A.A., Gridin V.V., Schechter I. // J. Phys. Chem. A. 1998. Vol. 102. N 47. P. 9470-9475. DOI: 10.1021/jp981942h

[2] Балакин А.А., Буйдо Е.А., Новикова Л.И. // ЖТФ. 2010. Т. 80. Вып. 9. С. 118-124. [Balakin A.A., Buido E.A., Novikova L.I. // Tech. Phys. 2010. Vol. 55. N 9. P. 1351-1356.] DOI: $10.1134 / \mathrm{S} 1063784210090197$

[3] Yakovlev B.S., Talrose V.L., Fenselau C. // Anal. Chem. 1994. Vol. 66. N 10. P. 1704-1707. DOI: 10.1021/ac00082a017

[4] Яковлев Б.С. // Химия высоких энергий. 1995. Т. 29. № 6. C. 421-422. [Yakovlev B.S. // High Energy Chemistry. 1995. Vol. 29. N 6. P. $389-390$.]

[5] Balakin A.A., Buido E.A., Golcova E.A., Novikova L.I., Talrose V.L. // J. Electrostat. 2006. Vol. 64. N 7-9. P. 555-561. DOI:10.1016/j.elstat.2005.10.010

[6] Балакин А.А., Буйдо Е.А. // ЖТФ. 2013. Т. 83. Вып. 11. C. 130-135. [Balakin A.A., Buido E.A. // Tech. Phys. 2013. Vol. 58. N 11. P. 1675-1680.] DOI: $10.1134 / \mathrm{S} 1063784213110042$

[7] Балакин А.А., Хидиров С.Г., Буйдо Е.А. // ЖТФ. 2016. Т. 86. Вып. 10. C. 32-37. [Balakin A.A., Khidirov S.G., Buido E.A. // Tech. Phys. 2016. Vol. 61. N 10. P. 1474-1479.] DOI: $10.1134 / \mathrm{S} 1063784216100054$

[8] Балакин А.А., Буйдо Е.А. // ЖТФ. 2018. Т. 88. Вып. 8. C. 1160-1163. DOI: 10.21883/JTF.2019.12.48500.452-18 [Balakin A.A., Buido E.A. // Tech. Phys. 2018. Vol. 63. N 8. P. 1125-1128.] DOI: $10.1134 / \mathrm{S} 1063784218080029$ 